\title{
Towards integrating information of service level agreement and resources as a services (RaaS) for cloud computing environment
}

\begin{abstract}
Cloud computing describes services and applications that are extended to be accessible through the Internet. Service Level Agreement (SLA) is a contractual agreement that has been established between the members of Cloud Service Provider (CSP) that consists of the service provider and its recipient for a specific application of services based on its allocating and sharing Resources as a Service (RaaS). In this context, there are many parties who are very concerning about it, but unfortunately there is lack of a common mapping of SLA information and RaaS of best practice as a guideline that can be used by CSP in making decision for the future purposes in engaging with services in the cloud computing environment. Therefore, the main goal of this paper is to integrate information of SLA and $\mathrm{RaaS}$ in order to ensure everybody who are providing and receiving the services in the cloud particularly will be satisfied and getting the best maximum Return Of the Investment (ROI) in allocating and sharing resources among the providers and recipients in cloud computing environment. As a result, some attributes such as reliability, readability and standardization of Software as a Service (SaaS), Platform as a Service (PaaS) and Infrastructure as a Service (IaaS) are found to be high.
\end{abstract}

Keyword: Cloud computing; Cloud service provider; Service level agreement; Resource allocation; Integrating information; Software as a service; Infrastructure as a service; Platform as a service 